\title{
BMJ Open Outcomes of upper gastrointestinal cancer screening in high-risk individuals: a population-based prospective study in Northeast China
}

\author{
Zhifu Yu, ${ }^{1}$ Tingting Zuo, ${ }^{1}$ Huihui Yu, ${ }^{1}$ Ying Zhao, ${ }^{2}$ Yong Zhang, ${ }^{3}$ Jinghua Liu, ${ }^{4}$ \\ Shoulan Dong, ${ }^{5}$ Ying Wu, ${ }^{6}$ Yunyong Liu (D) ${ }^{1}$
}

To cite: Yu Z, Zuo T, Yu H, et al. Outcomes of upper gastrointestinal cancer screening in high-risk individuals: a populationbased prospective study in Northeast China. BMJ Open 2022;12:e046134. doi:10.1136/ bmjopen-2020-046134

- Prepublication history and additional supplemental material for this paper are available online. To view these files, please visit the journal online (http://dx.doi.org/10.1136/ bmjopen-2020-046134)

Received 23 October 2020 Accepted 07 January 2022

Check for updates

(C) Author(s) (or their employer(s)) 2022. Re-use permitted under CC BY-NC. No commercial re-use. See rights and permissions. Published by BMJ.

For numbered affiliations see end of article.

Correspondence to

Dr Yunyong Liu;

liuyycancer@sina.com

\section{ABSTRACT}

Objectives The strategy for upper gastrointestinal cancer (UGC) screening has not yet been determined, especially in northeast China.

Design The sample was from an ongoing prospective population-based cohort for cancer screening.

Participants This study belonged to the Chinese Urban Cancer Screening Program. The analysis was based on the recruitment of participants aged 40-74 in Northeast China from 2016 to 2017. Totally, 39369 eligible participants were recruited, 8772 were evaluated to be at high risk for UGC, 1957 underwent endoscopy.

Outcomes $\chi^{2}$ test and multifactor logistic regression model was performed to analyse influencing factors of participation rate. Receiver operating characteristic curve analysis was applied to evaluate the diagnostic power of the high-risk assessment. The Cox regression model was used to estimate hazard ratio (HR) for the potential value. Results The high-risk rate was $22.28 \%$ and the participation rate of endoscopy screening was $22.31 \%$. Factors such as age at 45-59 years, female sex, high level of education, occupation for professional and technical personnel, former drinking, secondary smoking, less physical activity, history of trauma or mental depression, history of upper gastrointestinal system disease and family history of UGC were associated with increased participation in endoscopy screening (all the $p<0.05$ ). There were five UGCs, 86 oesophageal precancerous lesions and 145 gastric precancerous lesions, and the detection rates were $0.26 \%, 4.39 \%$ and $7.41 \%$, respectively. The detection rate for both oesophageal and gastric lesions increased with age and was higher for men than for women (all the $p<0.05$ ). After a 3-year follow-up, 30 UGCs had been diagnosed and the high risk of UGC increased the mortality risk ratio (HR: 1.90, $95 \%$ confidence interval (Cl) 1.41 to 2.56 ).

Conclusion The participation rate and outcomes of UGC screening were promising in our study and will provide important reference for evaluating value of UGC screening in China.

\section{INTRODUCTION}

Upper gastrointestinal cancer (UGC, including oesophageal cancer and gastric cancer) is one of the most commonly
Strengths and limitations of this study

- A prospective study was conducted under a national cancer screening programme and a passive method comparing with the population-based cancer registry and death registry to follow-up the current status.

- This high-risk group had relatively low compliance with endoscopic screening, and factors related to willingness to accept endoscopic screening had been identified.

- Endoscopy based on high-risk assessment was a feasible strategy for detecting upper gastrointestinal cancer and precursors.

- The risk factor questionnaire survey used to assess groups at high risk for upper gastrointestinal cancer needed to be improved.

- All such studies are limited by the quality of the questionnaire.

diagnosed cancers and leading causes of cancer-related deaths worldwide. In 2018, the number of new cases of oesophageal cancer was 572000 , ranking the seventh in the incidence of malignant tumours, and the number of deaths reached 509000 , ranking the sixth. Gastric cancer (including cardia cancer) is associated with more than one million new cases and 783000 deaths annually, being the fifth most common cancer and the third most common cause of cancer deaths. ${ }^{1}$ In China, oesophageal cancer and stomach cancer are the third and second most common malignancies, and mortality ranks the fourth and second, respectively. ${ }^{2}$ The resection rate of UGC in patients with advanced cancer is low and the prognosis is poor, with low survival rate, while early cancer can be completely removed by surgery and the 5-year survival rate is higher than $90 \% .{ }^{3}$ Studies have revealed that endoscopic screening holds great potential for reducing the burden of UGC. ${ }^{56}$ Until now, organised and opportunistic screening 
programmes have been widely carried out in many Asian countries such as Korea and Japan, which are high-income countries and typically have high incidence rates of UGC. ${ }^{78}$ Regarding the gold standard for UGC screening, endoscopy with biopsies for histopathological diagnosis is an invasive method requiring a high level of expertise, limiting its application in countries with intermediate or low incidence rates of UGC. Recently, the strategy of selecting high-risk patients for endoscopy through a risk stratification scoring system has been recommended in some aeras. ${ }^{9}{ }^{10}$ However, in population-based screening programmes, there is still little evidence to prove the effectiveness of this strategy that combines risk stratification and subsequent endoscopy.

The Chinese government has put a great deal of effort into UGC screening, including the Huaihe River Cancer Early Diagnosis and Treatment Project, a populationbased Cancer Screening Program in Rural China and a population-based Cancer Screening Program in Urban China (CanSPUC).

In our study, UGC cancer screening was conducted in Shenyang city in Liaoning province, China between October 2016 and August 2017 based on CanSPUC. In this project, eligible participants took a cancer risk assessment by an established Clinical Cancer Risk Score System first, and those who were evaluated as high risk for UGC were then recommended to undergo endoscopy at tertiary hospitals designated, free of charge. Our study aimed to assess the utility of the risk stratification and the feasibility of the endoscopy screening in high-risk individuals in northeast China and further to provide valid references for UGC screening strategy design in the future.

\section{METHODS}

\section{Study design and participants}

This was a population-based prospective study under the framework of CanSPUC, which was an ongoing national cancer screening programme in China. And a passive method comparing with the population-based cancer registry and death registry in Liaoning Province was used to follow-up the survival status of the populations until 30 September 2019. For the present analysis, we used the UGC screening data collected between October 2016 and August 2017 in Shenyang city (project implementation started in 2016) in Liaoning province. The cluster sampling method was used to select residents aged 40-74 years in 11 residential areas of Dadong district and 13 communities of Heping district of Shenyang city as the survey participants. All the participants approached by trained staff to complete a questionnaire in a face-to-face interview for high-risk assessment by a defined risk score system based on the Harvard Risk Index. ${ }^{11}$ The questionnaire mainly included general demographic characteristics, dietary habits (vegetables, fruits, dairy products, fried and hot foods, etc), lifestyle (smoking, drinking, drinking tea, etc), digestive tract history and family history of the disease (see online supplemental file 1). Participants who were evaluated as high risk of oesophageal cancer or gastric cancer were recommended to undergo endoscopy examination in Liaoning cancer hospital (tertiary hospitals in northeast China).

\section{Clinical procedures and outcome ascertainment}

All endoscopies were performed by experienced gastroenterologists (attending physician or above with at least 5 years of endoscopy experience). The abnormal findings during endoscopy were carefully examined in accordance with standard clinical procedures (endoscopy with indigo carmine dyeing and Lugol's iodine staining), and biopsy specimens were collected for further pathological diagnosis according to the clinical guidelines.

In this study, oesophageal precancerous lesion referred to squamous epithelial intraepithelial neoplasia or glandular intraepithelial neoplasia that occurred in the oesophagus; gastric precancerous lesion referred to glandular intraepithelial neoplasia that occurred in the stomach; oesophageal cancer referred to oesophageal squamous cell carcinoma adenocarcinoma, adenocarcinoma or other malignant tumours; gastric cancer referred to adenocarcinoma or other malignant tumours that occurred in the stomach.

\section{Quality control}

Strict and unified quality control standards were adopted in every step from the cancer risk assessment questionnaire to the clinical screening process, including the training of investigators, clinical screening technology training, the investigation information and screening data verifying. The endoscopy photo-documented collected during the screening process will be $100 \%$ review of images for positive cases and $1 \%$ review for negative cases by randomly selected.

\section{Statistical analysis}

The data were transmitted to the Central Data Management Team in the National Cancer Center of China where the database was constructed and analysis was performed. The EXCEL form was exported for collation, and then SPSS V.23.0 software was used for statistical analysis. If the measurement data had a normal distribution, they were expressed by the mean $\pm \mathrm{SD}(\mathrm{x} \pm \mathrm{S})$. Count data were expressed as a percentage $(\%)$, and comparisons between groups were tested by $\chi^{2}$ test. The analysis of influencing factors was performed by singlefactor analysis, and then the meaningful variables were incorporated into a binary multifactor logistic regression model. Sensitivity, specificity, Youden Index and receiver operating characteristic (ROC) curve analysis were used to evaluate the diagnostic power of the high-risk assessment based on the Harvard Risk Index. The sensitivity, specificity and Youden index were calculated as follows: sensitivity was equal to the number of patients with cancer who evaluated by high-risk assessment divided by the total number of patients with cancer; specificity was equal to the number of non-highrisk population by high-risk assessment divided by the total number of population without cancer; Youden index was equal to sensitivity plus specificity, then subtract 1 . The Cox 


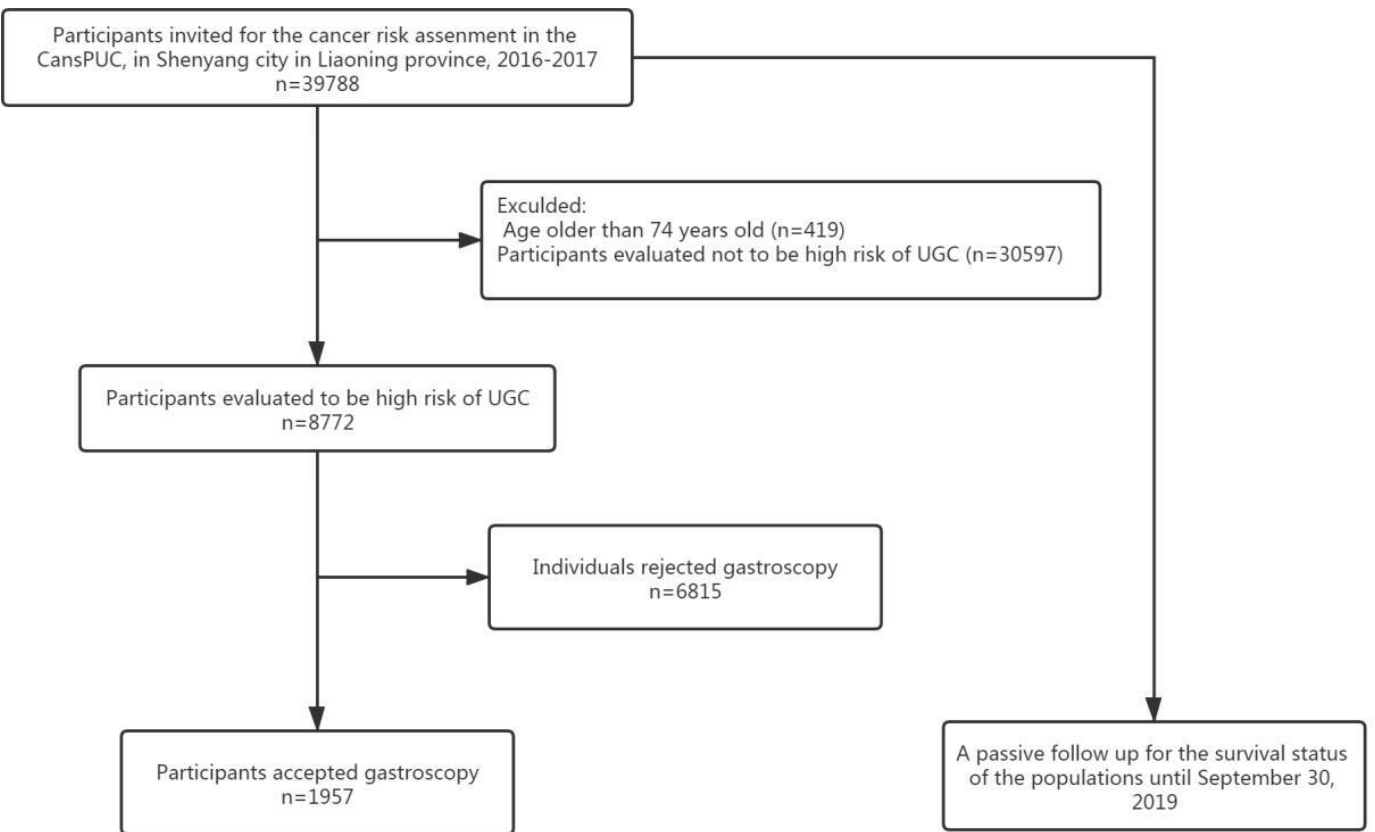

Figure 1 Flow diagram of participant recruitment. UGC, upper gastrointestinal cancer.

regression model was performed to compute the HR and 95\% CI for the potential value. All tests were two-sided and $p$ values of 0.05 or less were considered statistically significant.

\section{RESULTS}

Overall, 39788 participants were recruited. After excluding participants not at high risk for oesophageal cancer or gastric cancer $(\mathrm{N}=30597)$ and those ineligible on the basis of age $(\mathrm{N}=419)$, the final analysis included 8772 remaining participants at high UGC risk, among whom, 3313 were men and 5459 were women. A flowchart for the recruitment of the participants is shown in figure 1 .

\section{Risk assessment}

The high-risk rates stratified by age and sex were shown in table 1 . The high-risk rate was $22.28 \%$ and the rate of women $(24.06 \%)$ was higher than that of men $(19.86 \%)$ $\left(\chi^{2}=97.83, \mathrm{p}<0.001\right)$. The high-risk rate in different age groups was $17.06 \%$ at $40-44,22.49 \%$ at $45-49,23.44 \%$ at $49-54,25.29 \%$ at $55-59$ (the highest), $23.63 \%$ at $60-64,21.91 \%$ at $65-69$ and $15.36 \%$ at $70-74\left(\chi^{2}=194.87\right.$, $\mathrm{p}<0.001)$.

\section{Participation rate in endoscopy screening and its associated factors}

In total, 1957 participants at high risk for UGC completed clinical endoscopy examination, with a screening

\begin{tabular}{|c|c|c|c|c|c|}
\hline Factors & Assessment number & High risk of UGC & High-risk rates(\%) & $\chi^{2}$ value & $P$ value \\
\hline \multicolumn{6}{|c|}{ Age (years) } \\
\hline $40-44$ & 1934 & 330 & 17.06 & 194.87 & 0.000 \\
\hline $45-49$ & 4878 & 1097 & 22.49 & & \\
\hline $49-54$ & 5333 & 1250 & 23.44 & & \\
\hline $55-59$ & 7277 & 1840 & 25.29 & & \\
\hline $60-64$ & 8800 & 2079 & 23.63 & & \\
\hline $65-69$ & 7078 & 1551 & 21.91 & & \\
\hline $70-74$ & 4069 & 625 & 15.36 & & \\
\hline \multicolumn{6}{|l|}{ Sex } \\
\hline Male & 16680 & 3313 & 19.86 & 97.83 & 0.000 \\
\hline Female & 22689 & 5459 & 24.06 & & \\
\hline Total & 39369 & 8772 & 22.28 & & \\
\hline
\end{tabular}

UGC, upper gastrointestinal cancer. 
participation rate of $22.31 \%$. Among them, 1402 patients had a completed pathological biopsy, with a biopsy rate of $71.64 \%$. The participation rates stratified by potential associated factors were shown in table 2. Overall, the participation rate was higher in women than in men $(23.80 \%$ vs $19.86 \%, \mathrm{p}<0.001)$. The rate was highest among participants aged 45-49 years (26.98\%). Univariate analysis showed that participants who had a high educational level (27.74\% for undergraduate or over), worked as professional and technical personnel (28.72\%), were secondary smokers $(24.19 \%)$, were current or past alcohol drinkers $(23.15 \%$ vs $26.70 \%)$, lacked of physical activity $(23.81 \%)$, with trauma $(28.57 \%)$ or mental depression $(28.97 \%)$ history, had a history of upper gastrointestinal system disease $(23.74 \%)$ and had a family history of UGC (26.11\%) had relatively higher participation rates (all the $\mathrm{p}<0.05$ ).

Multivariable logistic regression analysis was also conducted to explore the potential factors that were associated with participation rate (see table 3 ). We found that people 40-69 years old, of female sex, with high level of education, secondary smoking, history of trauma or mental depression, history of upper gastrointestinal system disease and family history of UGC were associated with increased participation rate in endoscopy screening. The odds of a participant with a history of upper gastrointestinal system disease undertaking screening endoscopy were 1.77-fold higher than for a participant with no history of upper gastrointestinal system disease (OR: $1.77,95 \%$ CI 1.52 to 2.07 ). Similarly, the odds of a participant with a family history of UGC undertaking screening endoscopy were 1.37-fold higher than for a participant with no family history of UGC (OR: $1.37,95 \%$ CI 1.23 to $1.52)$.

\section{Upper gastrointestinal outcomes of endoscopy screening}

Table 4 showed the outcomes of endoscopy in our screening programme. Totally, there were five UGC, 86 oesophageal precancerous lesions (including squamous intraepithelial neoplasia and glandular intraepithelial neoplasia cases) and 145 gastric precancerous lesions (mainly cases of glandular intraepithelial neoplasm). The detection rates for UGC, oesophageal precancerous lesions and gastric precancerous lesions were $0.26 \%$, $4.39 \%$ and $7.41 \%$, respectively. The detection rates for oesophageal and gastric lesions increased with age, oesophageal lesions for men aged 49-59 years ranged from $2.04 \%$ to $4.05 \%$, significantly higher than the respective rate for women in the same age range (detection rate: $1.01 \%-3.58 \%$ ), and gastric lesions for men was significantly higher than for women in most age groups except for 49-54 years (detection rate for men: 5.06\%$21.26 \%$; detection rate for women: $3.03 \%-7.65 \%$ ) (all the $\mathrm{p}<0.05)$, as shown in figure 2 .

\section{Evaluation of the efficacy of the risk stratification for UGC}

To evaluate the efficacy of the risk stratification for UGC, we followed up the populations for 3 years, until
September 30, 2019. Ultimately, 30 participants were diagnosed with UGC, and the ratio was $0.14 \%$ in the high-risk population (12 vs 8760$)$ and $0.06 \%$ in the nonhigh-risk population ( 18 vs 30579$) \quad\left(\chi^{2}=5.44, \mathrm{p}=0.020\right)$. We found that those assessed as UGC high risk had an $90.00 \%$ increase in death risk when compared with those with lower risk (HR: $1.90,95 \%$ CI 1.41 to 2.56 ).

The sensitivity and specificity of the high-risk assessment based on the Harvard Risk Index were $40.00 \%$ and $77.72 \%$, respectively. The Youden Index was 0.18 . The area under the ROC curve (AUC) was 0.59 (95\% CI 0.46 to 0.70$)$, as shown in figure 3 .

\section{DISCUSSION}

It is well known that Japan, South Korea and China have a high incidence of UGC. ${ }^{12}$ Screening for UGC has been carried out in Japan from 1960 using gastrointestinal radiography to select positive cases of gastric cancer, and endoscopy is currently carried out nationwide for people aged 40 and above. South Korea has carried out nationwide upper gastrointestinal endoscopy screening once every 2 years for people aged 40 and above from 2002. We report the results of 1957 participants undergoing UGC screening in a population-based organised cancer screening programme in northeast China. In our study, the overall participation rate in UGC screening was $22.31 \%$, slightly higher than that for UGC screening in Henan province, China $(18.4 \%) .{ }^{13}$ The latter study demonstrated that factors such as male sex, high level of education, marriage, smoking, current alcohol drinking, lack of physical activity, history of upper gastrointestinal system disease and family history of UGC were associated with increased participation in endoscopy screening. Similarly, in our study, factors such as high educational background, professional and technical personnel, secondary smokers, current or past alcohol drinkers, lack of physical activity, history of trauma or mental depression, history of upper gastrointestinal system disease and family history of UGC were found to be associated with increased participation in endoscopy screening. Potential reasons for the lower rate in the previous study may include the invasiveness of endoscopy and insufficient knowledge of UGC screening. However, neither of the studies considered non-participants, who were not evaluated, and further study needs to be conducted. Therefore, in the case of low screening participation rates and low health awareness, we believe that extensive health education is an important measure to improve the effectiveness of screening.

The detection rates for oesophageal $(0.10 \%)$ and gastric $(0.15 \%)$ lesions were slightly higher than in Henan Province. ${ }^{13}$ The detection rates of both oesophageal and gastric cancer increased, consistent with that in Henan Province. The fluctuation of the detection rate in different genders and age groups might be related to the limited number of screening cases, different genders and 
Table 2 Factors associated with participation in endoscopy screening

\begin{tabular}{|c|c|c|c|c|c|}
\hline Factors & $\begin{array}{l}\text { Participants of high } \\
\text { risk for UGC }\end{array}$ & $\begin{array}{l}\text { Participants undertaking } \\
\text { gastroscopy }\end{array}$ & $\begin{array}{l}\text { Participation } \\
\text { rate }(\%)\end{array}$ & $\chi^{2}$ value & $P$ value \\
\hline \multicolumn{6}{|l|}{ Age (year) } \\
\hline $40-44$ & 330 & 75 & 22.73 & 57.29 & 0.000 \\
\hline $45-49$ & 1097 & 296 & 26.98 & & \\
\hline $49-54$ & 1250 & 285 & 22.80 & & \\
\hline $55-59$ & 1840 & 455 & 24.73 & & \\
\hline $60-64$ & 2079 & 466 & 22.41 & & \\
\hline $65-69$ & 1551 & 295 & 19.02 & & \\
\hline $70-74$ & 625 & 85 & 13.60 & & \\
\hline \multicolumn{6}{|l|}{ Sex } \\
\hline Male & 3313 & 658 & 19.86 & 18.84 & 0.000 \\
\hline Female & 5459 & 1299 & 23.80 & & \\
\hline \multicolumn{6}{|l|}{$\mathrm{BMI}$} \\
\hline$<18.5$ & 230 & 33 & 14.35 & 12.09 & 0.778 \\
\hline $18.5-23.9$ & 4205 & 972 & 23.12 & & \\
\hline $24-27.9$ & 3431 & 768 & 22.38 & & \\
\hline$\geq 28$ & 906 & 184 & 20.31 & & \\
\hline \multicolumn{6}{|l|}{ Education } \\
\hline Primary school or below & 336 & 54 & 16.07 & 58.69 & 0.000 \\
\hline Junior/senior school & 6075 & 1248 & 20.54 & & \\
\hline Undergraduate or over & 2361 & 655 & 27.74 & & \\
\hline \multicolumn{6}{|l|}{ Marriage } \\
\hline Unmarried & 741 & 181 & 24.43 & 2.09 & 0.148 \\
\hline Married & 8031 & 1776 & 22.11 & & \\
\hline \multicolumn{6}{|l|}{ Occupation } \\
\hline $\begin{array}{l}\text { Professional and technical } \\
\text { personnel }\end{array}$ & 1372 & 394 & 28.72 & 70.40 & 0.000 \\
\hline $\begin{array}{l}\text { Heads of state organs, } \\
\text { enterprises and institutions }\end{array}$ & 633 & 150 & 23.70 & & \\
\hline Staff & 1141 & 275 & 24.10 & & \\
\hline Businessmen & 301 & 68 & 22.59 & & \\
\hline Farmers & 172 & 28 & 16.28 & & \\
\hline Workers & 2711 & 506 & 18.66 & & \\
\hline Service workers & 557 & 132 & 23.70 & & \\
\hline Housework & 879 & 161 & 18.32 & & \\
\hline Other & 1006 & 243 & 24.16 & & \\
\hline \multicolumn{6}{|l|}{ Smoking } \\
\hline Never & 4914 & 1095 & 22.28 & 0.65 & 0.772 \\
\hline Current & 3320 & 749 & 22.56 & & \\
\hline Former & 538 & 113 & 21.00 & & \\
\hline \multicolumn{6}{|l|}{ Alcohol drinking } \\
\hline Never & 4273 & 903 & 21.13 & 9.02 & 0.011 \\
\hline Current & 4147 & 960 & 23.15 & & \\
\hline Former & 352 & 94 & 26.70 & & \\
\hline \multicolumn{6}{|l|}{ Second smoking } \\
\hline No & 2624 & 470 & 17.91 & 41.78 & 0.000 \\
\hline
\end{tabular}


Table 2 Continued

\begin{tabular}{|c|c|c|c|c|c|}
\hline Factors & $\begin{array}{l}\text { Participants of high } \\
\text { risk for UGC }\end{array}$ & $\begin{array}{l}\text { Participants undertaking } \\
\text { gastroscopy }\end{array}$ & $\begin{array}{l}\text { Participation } \\
\text { rate (\%) }\end{array}$ & $\chi^{2}$ value & $P$ value \\
\hline Yes & 6148 & 1487 & 24.19 & & \\
\hline \multicolumn{6}{|l|}{ Tea } \\
\hline Never & 5127 & 1136 & 22.16 & 0.92 & 0.632 \\
\hline Current & 3033 & 675 & 22.26 & & \\
\hline Former & 612 & 146 & 23.86 & & \\
\hline \multicolumn{6}{|l|}{ Physical activity } \\
\hline$<3$ times/week & 5805 & 1382 & 23.81 & 22.20 & 0.000 \\
\hline$\geq 3$ times/week & 2967 & 575 & 19.38 & & \\
\hline \multicolumn{6}{|l|}{ History of trauma } \\
\hline No & 5209 & 939 & 18.03 & 135.74 & 0.000 \\
\hline Yes & 3563 & 1018 & 28.57 & & \\
\hline \multicolumn{6}{|c|}{ History of mental depression } \\
\hline No & 5192 & 920 & 17.72 & 154.64 & 0.000 \\
\hline Yes & 3580 & 1037 & 28.97 & & \\
\hline \multicolumn{6}{|c|}{ History of upper gastrointestinal system disease } \\
\hline No & 1459 & 221 & 15.15 & 51.80 & 0.000 \\
\hline Yes & 7313 & 1736 & 23.74 & & \\
\hline \multicolumn{6}{|c|}{ Family history of UGC } \\
\hline No & 5283 & 1046 & 19.80 & 48.29 & 0.000 \\
\hline Yes & 3489 & 911 & 26.11 & & \\
\hline Total & 8772 & 1957 & 22.31 & & \\
\hline
\end{tabular}

BMI, body mass index; UGC, upper gastrointestinal cancer.

different participation rates in each age group. We would expand the sample size for future research.

The overall detection rate of oesophageal precancerous lesions was $4.39 \%$, higher than that of some populationbased screening programmes in China, but lower than that of some studies conducted in high-risk areas in other country. As reported, a screening programme by Zheng et al found that the overall detection rate of dysplasia was $1.57 \%$ for 12454 participants undertaking screening endoscopy in high-risk areas in rural China. ${ }^{14}$ In a population-based randomised controlled trial, 113 $(0.74 \%)$ individuals were diagnosed with high-grade oesophageal lesions. ${ }^{15}$ In another cross-sectional study that used 302 endoscopy screening records from highrisk areas in northern Iran, the overall dysplasia detection rate was $9.0 \% .^{16}$

The overall detection rate of gastric precancerous lesions was $7.41 \%$ in our study population, which was higher than for a population-based screening programme that used 924822 endoscopy screening records from Korea, with an overall detection rate for intraepithelial neoplasia of $4.2 \%{ }^{17}$ It should be noted that one of the reasons for the higher detection rate of upper gastrointestinal lesions in this study may have been the higher rate of pathological biopsy (71.64\%). Therefore, endoscopy and precision biopsy techniques in UGC screening should be improved.

Our study showed that factors such as age and sex had a positive association with oesophageal or gastric neoplasms. However, other sociodemographic factors including body mass index, smoking and drinking were not found to be associated with oesophageal or gastric neoplasms, which is not in line with previous studies. ${ }^{18} 19$ At the same time, a study by Hyo-Joon Yang et al revealed that diabetes mellitus was associated with an increased risk of gastric cancer, ${ }^{20}$ but there was no increase in the UGC detection rate in a population with a history of diabetes.

Existing studies in China have shown that carrying out endoscopic screening for UGC in high-risk rural areas can reduce the mortality associated with oesophageal cancer and gastric cancer, ${ }^{21}$ but the above-mentioned related studies have a small sample size and low level of evidence. Prospective randomised controlled studies aimed at evaluating the effect of UGC screening are very rare. ${ }^{15}$ The study by Chen et al revealed that one-time endoscopic screening among populations aged 40-69 years in high-risk areas of UGC could significantly decrease the incidence and mortality of UGC based on a multicentre population-based cohort study including 637500 people. ${ }^{22}$ Our study evaluated the screening results, and we found that the ratio diagnosed 
Table 3 Multivariable logistic regression models used to analyse the potential factors associated with participation rate

\begin{tabular}{|cll|}
\hline Factors & P value & OR (95\% Cl) \\
\hline Age (year) & & \\
\hline $40-44$ & 0.028 & 1.49 (1.04 to 2.13$)$ \\
$45-49$ & 0.000 & 1.81 (1.37 to 2.38$)$ \\
$49-54$ & 0.002 & 1.54 (1.17 to 2.02$)$ \\
$55-59$ & 0.000 & $1.78(1.37$ to 2.30$)$ \\
$60-64$ & 0.000 & $1.70(1.32$ to 2.20$)$ \\
$65-69$ & 0.009 & $1.43(1.09$ to 1.86$)$ \\
$70-74$ & Reference & \\
\hline
\end{tabular}

Sex

Male

Reference

Female

0.000

1.24 (1.11 to 1.38$)$

Education

Junior or below

Reference

Senior high school

0.410

$1.14(0.83$ to 1.56$)$

Undergraduate or over

0.028

1.45 (1.04 to 2.02$)$

Occupation

Professional and technical personnel

Heads of state organs, enterprises and institutions

Reference

Staff

0.098

0.83 (0.66 to 1.04$)$

Businessmen

0.014

0.79 (0.66 to 0.95$)$

Farmers

0.092

0.77 (0.57 to 1.04$)$

Workers

0.040

0.63 (0.40 to 0.98$)$

0.000

0.70 (0.59 to 0.83$)$

Service workers

0.075

0.80 (0.63 to 1.02$)$

Housework

0.000

0.61 (0.49 to 0.76$)$

other

0.100

0.843 (0.67 to 1.03$)$

Second smoking

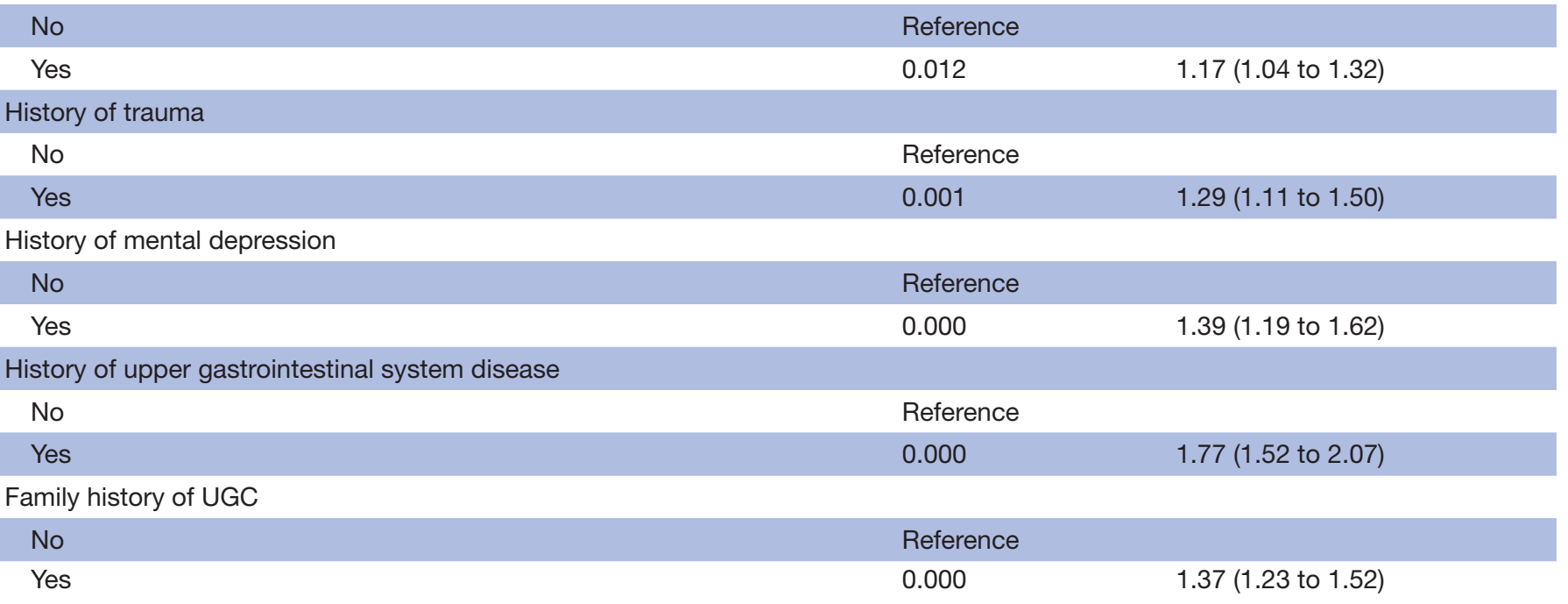

UGC, upper gastrointestinal cancer.

with UGC and death risk in participants at high UGC risk assessment were both higher than that in the non-high-risk population $(0.14 \%$ vs $0.06 \%, \chi 2=5.44, p=0.020$; HR: 1.90 , $95 \%$ CI 1.41 to 2.56 ). The high-risk assessment based on the Harvard Risk Index has a certain diagnostic power, as the sensitivity and specificity were $40.00 \%$ and $77.72 \%$, and the AUC was 0.59 (95\% CI 0.46 to 0.70). This demonstrates that the risk factor questionnaire survey results used to assess groups at high risk for UGC were acceptable. Due to the lack of objective quantitative indicators, the accuracy of 
Table 4 Oesophageal and gastric lesions detected by gastroscopy in the screening programme

\begin{tabular}{ll}
\hline Lesion & Detected number (rate\%) \\
\hline $\begin{array}{l}\text { Oesophageal precancerous } \\
\text { lesions }\end{array}$ & $86(4.39)$ \\
Gastric precancerous lesions & $145(7.41)$ \\
Oesophageal cancer & $2(0.10)$ \\
Gastric cancer & $3(0.15)$ \\
UGC & $5(0.26)$ \\
\hline
\end{tabular}

UGC, upper gastrointestinal cancer.

identifying the real high-risk groups needed to be improved, and the compliance of endoscopic screening was poor. As a result, the detection rate of upper gastrointestinal lesions was terminated. Therefore, in the follow-up project implementation, we are exploring to incorporate and objective methods, especially non-invasive early screening biomarkers, to improve feasibility of the screening strategies.

There were a few limitations in this study. First, the assessment of high-risk groups was based on questionnaires. Due to the subjective willingness of the respondents to undergo endoscopic screening, there might be biases in the information of the questionnaire to a certain extent. Second, the study mainly analysed the results of screeners in high-risk groups and did not analyse non-high-risk groups and could not evaluate the benefits of screening. Finally, this study was based on a prospective cohort, the follow-up time was relatively short at present, and the clear conclusions of the research were needed to be further studied.

\section{CONCLUSION}

In summary, the participation rate in this urban population-based UGC screening programme in northeast China was low. We identified several factors associated with the rate of participation in screening endoscopy

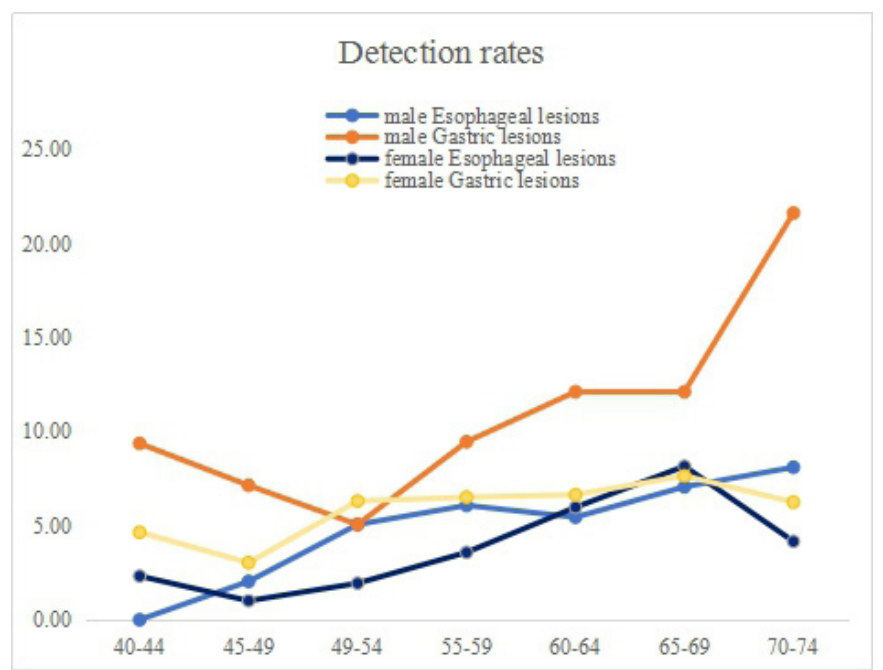

Figure 2 Detection rates of oesophageal lesions and gastric lesions, stratified by age and sex.

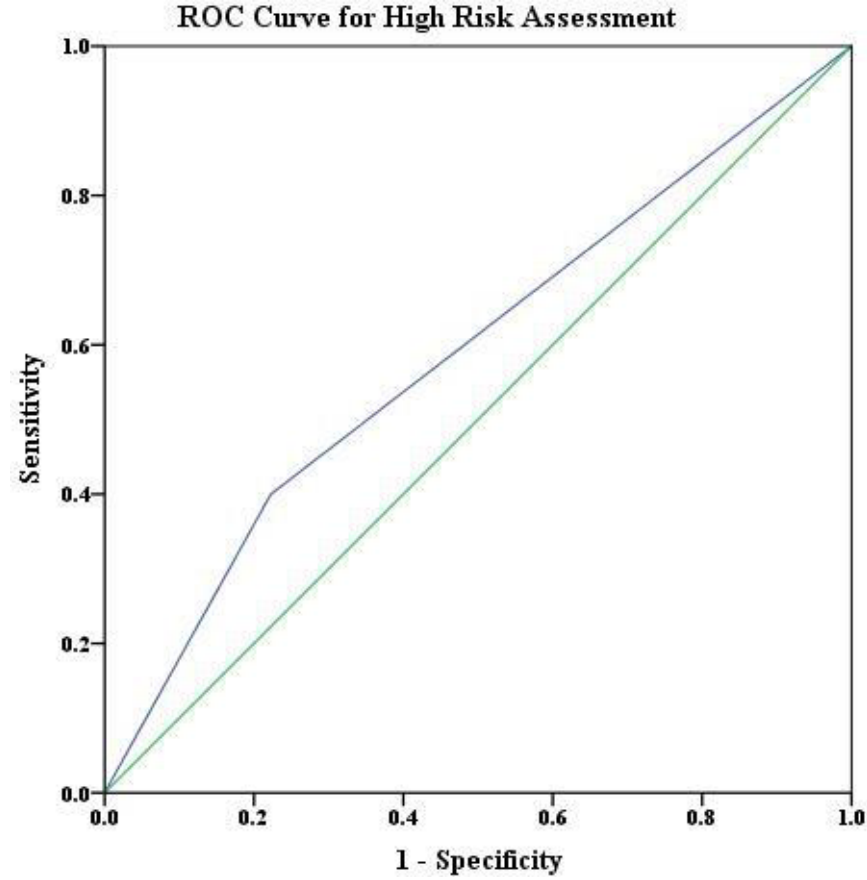

Figure 3 Evaluation of the power of the UGC highrisk assessment questionnaire. ROC, receiver operating characteristic; UGC, upper gastrointestinal cancer.

and there is room for improvement. The risk assessment and detection rate of UGC and precancerous lesions in our study were promising and will provide important references for designing efficacy population-based UGC screening strategies in the future.

\section{Author affiliations}

${ }^{1}$ Liaoning Office for Cancer Control and Research, Cancer Hospital of China Medical University, Liaoning Cancer Hospital and Institute, Shenyang, Liaoning, China

${ }^{2}$ Department of Endoscopy, Cancer Hospital of China Medical University, Liaoning Cancer Hospital and Institute, Shenyang, Liaoning, China

${ }^{3}$ Department of Pathology, Cancer Hospital of China Medical University, Liaoning Cancer Hospital and Institute, Shenyang, Liaoning, China

${ }^{4}$ Department of Radiology, Cancer Hospital of China Medical University, Liaoning Cancer Hospital and Institute, Shenyang, Liaoning, China

${ }^{5}$ Department of Chronic Non-Communicable Diseases, Heping District Center for Disease Control and Prevention, Shenyang, Liaoning, China

${ }^{6}$ Department of Chronic Non-Communicable Diseases, Dadong District Center for Disease Control and Prevention, Shenyang, Liaoning, China

Contributors $Z Y$ participated in analysis of data and drafting the manuscript. TZ participated in analysis of data. HY participated in controlling quality of the data. YiZ participated in screening of endoscopy. YoZ participated in pathological diagnosis. $\mathrm{JL}$ participated in screening of endoscopy. YW and SD participated in population recruitment. YL participated the design of the study and as guarantor of this article.

Funding This work was supported by Young and middle-aged scientific and technological talent support plan project, Shenyang city, China [RC200437] and Key Research and Development Programme project of Liaoning Province [2019JH2/10300013].

Competing interests None declared.

Patient consent for publication Consent obtained directly from patient(s).

Ethics approval This study involves human participants and was approved by the Ethics Committee of the National Cancer Center/Cancer Hospital, Chinese Academy of Medical Sciences and Peking Union Medical College and all participants provided a written informed consent. Approval Number 15-070/997. Participants gave informed consent to participate in the study before taking part. 
Provenance and peer review Not commissioned; externally peer reviewed.

Data availability statement Data are available upon reasonable request. All data used during the study are available from the corresponding author by request.

Supplemental material This content has been supplied by the author(s). It has not been vetted by BMJ Publishing Group Limited (BMJ) and may not have been peer-reviewed. Any opinions or recommendations discussed are solely those of the author(s) and are not endorsed by BMJ. BMJ disclaims all liability and responsibility arising from any reliance placed on the content. Where the content includes any translated material, BMJ does not warrant the accuracy and reliability of the translations (including but not limited to local regulations, clinical guidelines, terminology, drug names and drug dosages), and is not responsible for any error and/or omissions arising from translation and adaptation or otherwise.

Open access This is an open access article distributed in accordance with the Creative Commons Attribution Non Commercial (CC BY-NC 4.0) license, which permits others to distribute, remix, adapt, build upon this work non-commercially, and license their derivative works on different terms, provided the original work is properly cited, appropriate credit is given, any changes made indicated, and the use is non-commercial. See: http://creativecommons.org/licenses/by-nc/4.0/.

ORCID iD

Yunyong Liu http://orcid.org/0000-0002-5224-7828

\section{REFERENCES}

1 Ferlay J, Colombet M, Soerjomataram I, et al. Estimating the global cancer incidence and mortality in 2018: GLOBOCAN sources and methods. Int J Cancer 2019;144:1941-53.

2 Chen W, Zheng R, Baade PD, et al. Cancer statistics in China, 2015. CA Cancer J Clin 2016;66:115-32.

3 Wang G-Q, Jiao G-G, Chang F-B, et al. Long-term results of operation for 420 patients with early squamous cell esophageal carcinoma discovered by screening. Ann Thorac Surg 2004;77:1740-4.

4 Yang S, Wu S, Huang Y, et al. Screening for oesophageal cancer. Cochrane Database Syst Rev 2012;2.

5 Wei W-Q, Chen Z-F, He Y-T, et al. Long-Term follow-up of a community assignment, one-time endoscopic screening study of esophageal cancer in China. J Clin Oncol 2015;33:1951-7.

6 Matsumoto S, Ishikawa S, Yoshida Y. Reduction of gastric cancer mortality by endoscopic and radiographic screening in an isolated island: a retrospective cohort study. Aust $J$ Rural Health 2013;21:319-24.
$7 \mathrm{Kim}$ Y, Jun JK, Choi KS, et al. Overview of the National cancer screening programme and the cancer screening status in Korea. Asian Pac J Cancer Prev 2011;12:725-30.

8 Veitch AM, Uedo N, Yao K, et al. Optimizing early upper gastrointestinal cancer detection at endoscopy. Nat Rev Gastroenterol Hepatol 2015;12:660-7.

9 Rubenstein JH, Thrift AP. Risk factors and populations at risk: selection of patients for screening for Barrett's oesophagus. Best Pract Res Clin Gastroenterol 2015;29:41-50.

10 Kunzmann AT, Thrift AP, Cardwell CR, et al. Model for identifying individuals at risk for esophageal adenocarcinoma. Clin Gastroenterol Hepatol 2018;16:1229-36.

11 Grosso G, Bella F, Godos J, et al. Possible role of diet in cancer: systematic review and multiple meta-analyses of dietary patterns, lifestyle factors, and cancer risk. Nutr Rev 2017;75:405-19.

12 Leung WK, Wu M-shiang, Kakugawa Y, et al. Screening for gastric cancer in Asia: current evidence and practice. Lancet Oncol 2008;9:279-87.

13 Guo L, Zhang S, Liu S, et al. Determinants of participation and detection rate of upper gastrointestinal cancer from populationbased screening program in China. Cancer Med 2019;8:7098-107.

14 Zheng X, Mao X, Xu K, et al. Massive endoscopic screening for esophageal and gastric cancers in a high-risk area of China. PLoS One 2015;10:e0145097.

$15 \mathrm{He} \mathrm{Z}$, Liu Z, Liu M, et al. Efficacy of endoscopic screening for esophageal cancer in China (ESECC): design and preliminary results of a population-based randomised controlled trial. Gut 2019;68:198-206.

16 Roshandel G, Khoshnia M, Sotoudeh M, et al. Endoscopic screening for precancerous lesions of the esophagus in a high risk area in northern Iran. Arch Iran Med 2014;17:246-52.

17 Choi KS, Jun JK, Park E-C, et al. Performance of different gastric cancer screening methods in Korea: a population-based study. PLOS One 2012;7:e50041.

18 Dong J, Thrift AP. Alcohol, smoking and risk of oesophago-gastric cancer. Best Pract Res Clin Gastroenterol 2017;31:509-17.

19 Kim YM, Kim J-H, Baik SJ, et al. Sarcopenia and sarcopenic obesity as novel risk factors for gastric carcinogenesis: a health checkup cohort study. Front Oncol 2019;9:1249.

20 Yang $\mathrm{H}-\mathrm{J}$, Kang D, Chang Y, et al. Diabetes mellitus is associated with an increased risk of gastric cancer: a cohort study. Gastric Cancer 2020;23:382-90.

21 Chen Q, Yu L, Hao C, et al. Effectiveness evaluation of organized screening for esophageal cancer: a case-control study in Linzhou City, China. Sci Rep 2016;6:35707.

22 Chen R, Liu Y, Song G, et al. Effectiveness of one-time endoscopic screening programme in prevention of upper gastrointestinal cancer in China: a multicentre population-based cohort study. Gut 2021;70:251-60. 